Research Article

\title{
Homotopy Perturbation Method for Solving Wave-Like Nonlinear Equations with Initial-Boundary Conditions
}

\begin{abstract}
Afgan Aslanov
Mathematics and Computing Department, Beykent University, 34396 Istanbul, Turkey

Correspondence should be addressed to Afgan Aslanov, afganaslanov@beykent.edu.tr

Received 4 May 2011; Revised 27 June 2011; Accepted 20 July 2011

Academic Editor: Pham Huu Anh Ngoc

Copyright ( $) 2011$ Afgan Aslanov. This is an open access article distributed under the Creative Commons Attribution License, which permits unrestricted use, distribution, and reproduction in any medium, provided the original work is properly cited.

The homotopy perturbation method is employed to obtain approximate analytical solutions of the wave-like nonlinear equations with initial-boundary conditions. An efficient way of choosing the auxiliary operator is presented. The results demonstrate reliability and efficiency of the method.
\end{abstract}

\section{Introduction}

In this paper, we consider the equation

$$
u_{t t}-c^{2} u_{x x}=f\left(x, t, u, u_{x}, u_{y}, u_{x t}\right)+g(x, t), \quad 0<x<\infty
$$

with initial conditions

$$
u(x, 0)=\varphi(x), \quad u_{t}(x, 0)=\psi(x),
$$

and boundary condition

$$
u(0, t)=h(t),
$$

where $f, g, \varphi, \psi$, and $h$ are known functions.

Problems like (1.1)-(1.2)-(1.3) model many problems in classical and quantum mechanics, solitons, and matter physics $[1,2]$. If $f$ is a function of $u$ only, we obtain a KleinGordon or sine-Gordon-type equations. 
In the last decade, some various approximate methods have been developed, such as the homotopy perturbation method (HPM) [3-13] and Adomian's decomposition method (ADM) [14-20] to solve linear and nonlinear differential equations.

Unlike the various approximation techniques for solving nonlinear wave type problems, which are usually valid for initial value problems (without boundary conditions) or some special type of problems (homogenous, etc.), our technique is applicable for all initial-boundary problems of type (1.1)-(1.2)-(1.3). Chowdhury and Hashim [9] applied the HPM for solving Klein-Gordon and sine-Gordon equations, with initial conditions (1.2). El-Sayed [19] and Wazwaz and Gorguis [20] used ADM for solving wave-like and heatlike problems. Their approaches cannot be applied for all wave-like equations with initialboundary conditions since the operator $L=u_{t t}$ cannot control the boundary condition (1.3) see Example 2.1 below.

The central idea here is that the problem

$$
\begin{aligned}
u_{t t}-c^{2} u_{x x} & =g(x, t), \\
u(x, 0)=\varphi(x), \quad u_{t}(x, 0) & =\psi(x), \quad u(0, t)=h(t),
\end{aligned}
$$

has a unique solution (see, e.g., [21]) and therefore there exists an inverse of the operator $L=u_{t t}-c^{2} u_{x x}$.

The main idea of HPM is to introduce a homotopy parameter, say $p$, which takes values from 0 to 1 . When $p=0$ the equation usually reduces to a sufficiently simplified form (linear or very easy nonlinear). As $p$ increases to 1 , the equation goes through a sequence of "deformations" (homotopics) and at $p=1$ takes the original form of the equation.

We rewrite (1.1) as

$$
L u=f\left(x, t, u, u_{x}, u_{y}, u_{x y}\right)+g(x, t),
$$

and construct the following homotopy:

$$
L u-L v_{0}+p\left[L v_{0}-f\left(x, t, u, u_{x}, u_{y}, u_{x y}\right)-g(x, t)\right]=0
$$

Usually we take $v_{0}$ as a solution of the problem (1.1)-(1.2)-(1.3) with $f=0$ or simply $v_{0}=0$. Assume that the solution of (1.6) is in the form

$$
u=u_{0}+p u_{1}+p u_{2}+\cdots,
$$

and substituting (1.7) into (1.6) and equating terms of the same powers of $p$ we obtain a system of equations for $u_{0}, u_{1}, u_{2}, \ldots$. Solving these system of equations we obtain a solution in the form

$$
u=u_{0}+u_{1}+u_{2}+\cdots
$$




\section{Applications}

The HPM and ADM offer excellent choices for obtaining the closed-form analytical solutions of wave-like equations. Chowdhury and Hashim [9], El-Sayed [19], and Wazwaz and Gorguis [20] recently showed how the HPM and ADM can be applied to find an analytic approximate solution of wave-like equations with initial conditions (1.2). They mainly used the operator $L=u_{t t}$ for solving the wave-like problems. But in case of inhomogeneous nonlinear or even linear equations with initial-boundary conditions, these approaches have some difficulties. If we construct the standard homotopy with $L=u_{t t}$, for solving wave-like equations with initial-boundary conditions, usually in the second or even in the first stage of HPM, we obtain an "overdetermined" or very difficult problem. To explain these difficulties we consider the following example.

Example 2.1. Consider the linear problem

$$
\begin{aligned}
u_{t t}-u_{x x} & =6 x, \\
u(x, 0)=-x^{3}, \quad & u_{t}(x, 0)=0, \quad u(0, t)=\sin ^{2} t .
\end{aligned}
$$

The exact solution is

$$
\begin{aligned}
u & =-x^{3}+\sin ^{2}(t-x) \text { for } x<t \\
& =-x^{3} \quad \text { for } x>t
\end{aligned}
$$

Using our HPM technique we can easily find this solution. Indeed, let us take $L=u_{t t}-u_{x x}$, $v_{0}=0$ and construct the homotopy

$$
L u-L v_{0}+p\left[L v_{0}-6 x\right]=0 .
$$

Now substituting (1.7) into $u$ and equating the coefficients of like powers of $p$, we get a system of linear equations

$$
\begin{array}{rrrr}
L u_{0}=0, & u_{0}(x, 0)=-x^{3}, & u_{0 t}(x, 0)=0, & u_{0}(0, t)=\sin ^{2} t, \\
L u_{1}=6 x, & u_{1}(x, 0)=0, & u_{1 t}(x, 0)=0, & u_{1}(0, t)=0, \\
L u_{2}=0, & u_{2}(x, 0)=0, \quad u_{2 t}(x, 0)=0, & u_{2}(0, t)=0, \ldots
\end{array}
$$


Solving correspondingly we obtain

$$
\begin{aligned}
u_{0} & =-\frac{1}{2}\left((x+t)^{3}+(x-t)^{3}\right)=-3 t^{2} x-x^{3} \text { for } x \geq t \\
& =-3 t^{2} x-x^{3}+\sin ^{2}(t-x) \text { for } x<t, \\
u_{1} & =\frac{1}{2} \int_{0}^{t} \int_{x-t+s}^{x+t-s} 6 y d y d s=3 t^{2} x, \text { for } x>t, \\
u_{1} & =\frac{1}{2} \int_{0}^{t-x} \int_{t-x-s}^{x+t-s} 6 y d y d s+\frac{1}{2} \int_{t-x}^{t} \int_{x-t+s}^{x+t-s} 6 y d y d s=3 t^{2} x, \text { for } x<t .
\end{aligned}
$$

$u_{2}=0, \ldots$. Thus we obtain an exact solution $u_{\text {exact }}=u_{1}+u_{2}$.

Now let us show that this problem can not be solved when $L$ is taken as $L=u_{t t}$.

Indeed if we choose $v_{0}=0$ or $v_{0}=-x^{3}$ (which seems most natural and appropriate) and $L=u_{t t}$ and construct the homotopy

$$
L u-L v_{0}+p\left[L v_{0}-u_{x x}-6 x\right]=0
$$

we obtain an overdetermined problem for $u_{0}$ in the form

$$
L u_{0}=0, \quad u_{0}(x, 0)=-x^{3}, \quad u_{0 t}(x, 0)=0, \quad u_{0}(0, t)=\sin ^{2} t
$$

which has no solution.

If in the first stage we consider the boundary conditions $u_{0}(x, 0)=-x^{3}, u_{0 t}(x, 0)=0$, $u_{0}(0, t)=0$, we obtain $u_{0}=-x^{3}$ and in the second stage we need to solve the problem

$$
L u_{1}=u_{0 x x}+6 x, \quad u_{1}(x, 0)=0, \quad u_{1 t}(x, 0)=0, \quad u_{1}(0, t)=\sin ^{2} t,
$$

which is overdetermined again and has no solution.

Example 2.2. Consider the problem

$$
\begin{gathered}
u_{t t}-u_{x x}=u_{x} u-e^{2 t} x+e^{t} x, \\
u(x, 0)=x, \quad u_{t}(x, 0)=x, \quad u(0, t)=0 .
\end{gathered}
$$

The exact solution is $u=e^{t} x$. We take again $L=u_{t t}-u_{x x}, v_{0}=0$, and construct the homotopy

$$
L u-L v_{0}+p\left[L v_{0}-u_{x} u+e^{2 t} x-e^{t} x\right]=0
$$


Substituting (1.7) into (2.10) we obtain

$$
\begin{aligned}
& L\left(u_{0}+p u_{1}+p^{2} u_{2}+\cdots\right)-L v_{0} \\
& \quad+p\left[L v_{0}-\left(u_{0 x}+p u_{1 x}+p^{2} u_{2 x}+\cdots\right)\left(u_{0}+p u_{1}+\cdots\right)+e^{2 t} x-e^{t} x\right]=0
\end{aligned}
$$

and equating terms of the coefficients of like powers of $p$ gives

$$
\begin{gathered}
L u_{0}=L v_{0}, \quad u_{0}(x, 0)=x, \quad u_{0 t}(x, 0)=x, \quad u_{0}(0, t)=0, \\
L u_{1}+L v_{0}-u_{0 x} u_{0}+e^{2 t} x-e^{t} x=0, \quad u_{1}(x, 0)=0, \quad u_{1 t}(x, 0)=0, \quad u_{1}(0, t)=0, \\
L u_{2}-u_{0 x} u_{1}-u_{1 x} u_{0}=0, \quad u_{2}(x, 0)=0, \quad u_{2 t}(x, 0)=0, \quad u_{2}(0, t)=0, \\
L u_{3}-u_{2 x} u_{0}-u_{1 x} u_{1}-u_{0 x} u_{2}=0, \quad u_{3}(x, 0)=0, \quad u_{3 t}(x, 0)=0, \quad u_{3}(0, t)=0, \ldots
\end{gathered}
$$

Solving these equations we obtain (see [21, Chapter 3.4])

$$
\begin{gathered}
u_{0}=\frac{1}{2}[(x+t)+(x-t)]+\frac{1}{2} \int_{x-t}^{x+t} y d y=x+\frac{(x+t)^{2}}{4}-\frac{(x-t)^{2}}{4}=x(t+1), \\
u_{1}=\frac{1}{2} \int_{0}^{t} \int_{x-t+s}^{x+t-s}\left(y(s+1)^{2}-e^{2 s} y+e^{s} y\right) d y d s=\frac{x}{12}\left(t^{4}+4 t^{3}+6 t^{2}-6 t-3 e^{2 t}+12 e^{t}-9\right),
\end{gathered}
$$

for $x>t$ and

$$
\begin{aligned}
u_{1}= & \frac{1}{2} \int_{0}^{t-x} \int_{t-x-s}^{x+t-s} g(y, s) d y d s+\frac{1}{2} \int_{t-x}^{t} \int_{x-t+s}^{x+t-s} g(y, s) d y d s \\
= & x^{2} e^{t-x}-\frac{1}{4} x e^{2 t-2 x}-\frac{3}{4} x+\frac{1}{2} t^{2} x-t x^{3}+\frac{1}{3} t^{3} x+\frac{2}{3} t x^{4}+\frac{1}{12} t^{4} x-\frac{1}{2} x^{2} e^{2 t-2 x} \\
& +x e^{t-x}-\frac{1}{2} t^{2} x^{3}-\frac{1}{2} t x-\frac{1}{2} x^{3}+\frac{2}{3} x^{4}-\frac{1}{4} x^{5}+t x^{3}-\frac{2}{3} t x^{4}-\frac{1}{4} x e^{2 t}+\frac{1}{2} t^{2} x^{3} \\
& +x e^{t}+\frac{1}{2} x^{3}-\frac{2}{3} x^{4}+\frac{1}{4} x^{5}+\frac{1}{2} x^{2} \frac{e^{2 t}}{e^{2 x}}-x \frac{e^{t}}{e^{x}}-x^{2} \frac{e^{t}}{e^{x}}+\frac{1}{4} x \frac{e^{2 t}}{e^{2 x}} \\
= & \frac{1}{12} x t^{4}+\frac{1}{3} x t^{3}+\frac{1}{2} x t^{2}-\frac{1}{2} x t-\frac{1}{4} x e^{2 t}+x e^{t}-\frac{3}{4} x
\end{aligned}
$$


Table 1: Maximum errors for Example 2.2.

\begin{tabular}{ccccccccc}
\hline$t \backslash x$ & 0.1 & 0.2 & 0.3 & 0.4 & 0.5 & 0.6 & 0.8 & 0.9 \\
\hline 0.1 & $1 \times 10^{-9}$ & $3 \times 10^{-9}$ & $4 \times 10^{-9}$ & $6 \times 10^{-9}$ & $7 \times 10^{-9}$ & $9 \times 10^{-9}$ & $10^{-8}$ & $10^{-8}$ \\
0.2 & $3 \times 10^{-9}$ & $2 \times 10^{-7}$ & $3 \times 10^{-7}$ & $4 \times 10^{-7}$ & $5 \times 10^{-7}$ & $6 \times 10^{-7}$ & $8 \times 10^{-7}$ & $9 \times 10^{-7}$ \\
0.4 & $6 \times 10^{-9}$ & $4 \times 10^{-7}$ & $5 \times 10^{-6}$ & $3 \times 10^{-5}$ & $4 \times 10^{-5}$ & $4 \times 10^{-5}$ & $6 \times 10^{-5}$ & $7 \times 10^{-5}$ \\
0.5 & $7 \times 10^{-9}$ & $5 \times 10^{-7}$ & $6 \times 10^{-6}$ & $4 \times 10^{-5}$ & $10^{-4}$ & $2 \times 10^{-4}$ & $2 \times 10^{-4}$ & $3 \times 10^{-4}$ \\
0.7 & $10^{-8}$ & $7 \times 10^{-7}$ & $9 \times 10^{-6}$ & $5 \times 10^{-5}$ & $2 \times 10^{-4}$ & $7 \times 10^{-4}$ & $2 \times 10^{-3}$ & $2 \times 10^{-3}$ \\
0.8 & $7 \times 10^{-4}$ & $10^{-3}$ & $2 \times 10^{-3}$ & $3 \times 10^{-3}$ & $3 \times 10^{-3}$ & $4 \times 10^{-3}$ & $5 \times 10^{-3}$ & $6 \times 10^{-3}$ \\
1.0 & $3 \times 10^{-3}$ & $6 \times 10^{-3}$ & $9 \times 10^{-3}$ & $10^{-2}$ & $10^{-2}$ & 0.0184 & $2 \times 10^{-2}$ & $3 \times 10^{-2}$ \\
\hline
\end{tabular}

for $x<t$. In a similar manner we have

$$
\begin{aligned}
L u_{2}= & u_{0 x} u_{1}+u_{1 x} u_{0}=(t+1) \frac{1}{12} x\left(t^{4}+4 t^{3}+6 t^{2}-6 t-3 e^{2 t}+12 e^{t}-9\right) \\
& +\frac{1}{12}\left(t^{4}+4 t^{3}+6 t^{2}-6 t-3 e^{2 t}+12 e^{t}-9\right)(t+1) x \\
= & \frac{1}{6} x(t+1)\left(t^{4}+4 t^{3}+6 t^{2}-6 t-3 e^{2 t}+12 e^{t}-9\right),
\end{aligned}
$$

and therefore

$$
\begin{aligned}
u_{2} & =L^{-1}\left(\frac{1}{6} x(t+1)\left(t^{4}+4 t^{3}+6 t^{2}-6 t-3 e^{2 t}+12 e^{t}-9\right)\right) \\
& =\frac{1}{2} \int_{0}^{t} \int_{x-t+s}^{x+t-s}\left(\frac{1}{6} y(s+1)\left(s^{4}+4 s^{3}+6 s^{2}-6 s-3 e^{2 s}+12 e^{s}-9\right)\right) d y d s \\
& =\frac{x}{504}\left(2 t^{7}+14 t^{6}+42 t^{5}-210 t^{3}-378 t^{2}-63 t e^{2 t}+1008 t e^{t}+63 t-1008 e^{t}+1008\right),
\end{aligned}
$$

for $x>t$. In a similar manner we obtain

$$
\begin{aligned}
L u_{2} & =u_{0 x} u_{1}+u_{1 x} u_{0}=\frac{x t^{5}}{6}+\frac{5}{6} x t^{4}+\frac{5}{3} x t^{3}-\frac{x t e^{2 t}}{2}+2 x t e^{t}-\frac{5 x t}{2}-\frac{x e^{2 t}}{2}+2 x e^{t}-\frac{3 x}{2} \\
u_{2} & =\frac{1}{2} \int_{0}^{t-x} \int_{t-x-s}^{x+t-s} g(y, s) d y d s+\frac{1}{2} \int_{t-x}^{t} \int_{x-t+s}^{x+t-s} g(y, s) d y d s
\end{aligned}
$$

for $x<t$, where $g(y, s)=(1 / 6) y s^{5}+(5 / 6) y s^{4}+(5 / 3) y s^{3}-(1 / 2) y s e^{2 s}+2 y s e^{s}-(5 / 2) y s-$ $(1 / 2) y e^{2 s}+2 y e^{s}-(3 / 2) y$ and so

$$
u_{2}=\frac{1}{252} x t^{7}+\frac{1}{36} x t^{6}+\frac{1}{12} x t^{5}-\frac{5}{12} x t^{3}-\frac{3}{4} x t^{2}-\frac{1}{8} x t e^{2 t}+2 x t e^{t}+\frac{1}{8} x t-2 x e^{t}+2 x .
$$

The absolute errors between the exact and three term approximation of the series solution for some values of $(x, t) \in[0,1] \times[0,1]$ are shown in Table 1 . 
Discrete Dynamics in Nature and Society

Example 2.3. Now we consider the problem

$$
\begin{gathered}
u_{t t}-u_{x x}=u_{x t}^{2}-16 t^{2} x^{2}+16 t^{2} x-2 t^{2}-2 x^{2}+2 x, \\
u(x, 0)=x, \quad u_{t}(x, 0)=0, \quad u(0, t)=0 .
\end{gathered}
$$

The exact solution is $u=x+t^{2} x-t^{2} x^{2}$. We take $v_{0}=0$, and construct the homotopy

$$
L u-L v_{0}+p\left[L v_{0}-u_{x t}^{2}+16 t^{2} x^{2}-16 t^{2} x+2 t^{2}+2 x^{2}-2 x\right]=0
$$

Now substituting (1.7) into (2.20) and equating terms of the coefficients of like powers of $p$, we obtain

$$
\begin{gathered}
L u_{0}=0, \quad u_{0}(x, 0)=x, \quad u_{0 t}(x, 0)=u_{0}(0, t)=0, \\
L u_{1}+L v_{0}-u_{0 x t}^{2}+16 t^{2} x^{2}-16 t^{2} x+2 t^{2}+2 x^{2}-2 x=0, \quad u_{1}(x, 0)=u_{1 t}(x, 0)=u_{1}(0, t)=0, \\
L u_{2}-2 u_{0 x t} u_{1 x t}=0, \quad u_{2}(x, 0)=u_{2 t}(x, 0)=u_{2}(0, t)=0, \\
L u_{3}-\left(u_{1 x t}\right)^{2}-2 u_{0 x t} u_{2 x t}=0, \quad u_{3}(x, 0)=u_{3 t}(x, 0)=u_{3}(0, t)=0 .
\end{gathered}
$$

Solving equations yields

$$
\begin{aligned}
u_{0} & =\frac{1}{2}(x+t+x-t)=x \\
u_{1} & =\frac{1}{2} \int_{0}^{t} \int_{x-t+s}^{x+t-s}\left(-16 s^{2} y^{2}+16 s^{2} y-2 s^{2}-2 y^{2}+2 y\right) d y d s \\
& =-\frac{4}{45} t^{6}-\frac{4}{3} t^{4} x^{2}+\frac{4}{3} t^{4} x-\frac{1}{3} t^{4}-t^{2} x^{2}+t^{2} x \quad \text { for } x>t .
\end{aligned}
$$

In a similar manner we get

$$
\begin{aligned}
u_{1}= & \frac{1}{2} \int_{0}^{t-x} \int_{t-x-s}^{x+t-s}\left(-16 s^{2} y^{2}+16 s^{2} y-2 s^{2}-2 y^{2}+2 y\right) d y d s \\
& +\frac{1}{2} \int_{t-x}^{t} \int_{x-t+s}^{x+t-s}\left(-16 s^{2} y^{2}+16 s^{2} y-2 s^{2}-2 y^{2}+2 y\right) d y d s=-\frac{8}{15} t^{5} x-\frac{4}{3} t^{4} x \\
& -\frac{16}{9} t^{3} x^{3}-\frac{4}{3} t^{3} x+\frac{4}{3} t^{2} x^{4}+t^{2} x^{2}+t^{2} x-\frac{8}{15} t x^{5}-\frac{4}{3} t x^{3}+\frac{4 x^{6}}{45}+\frac{x^{4}}{3}
\end{aligned}
$$


Table 2: Maximum errors for Example 2.3.

\begin{tabular}{cccccccc}
\hline$t \backslash x$ & 0.1 & 0.2 & 0.4 & 0.5 & 0.7 & 0.8 & 0.9 \\
\hline 0.1 & $2 \times 10^{-5}$ & $10^{-5}$ & $10^{-6}$ & $9 \times 10^{-8}$ & $5 \times 10^{-6}$ & $10^{-5}$ & $2 \times 10^{-5}$ \\
0.2 & $3 \times 10^{-4}$ & $2 \times 10^{-4}$ & $2 \times 10^{-5}$ & $5 \times 10^{-6}$ & $9 \times 10^{-5}$ & $2 \times 10^{-4}$ & $3 \times 10^{-4}$ \\
0.4 & $3 \times 10^{-3}$ & $3 \times 10^{-3}$ & $7 \times 10^{-4}$ & $3 \times 10^{-4}$ & $2 \times 10^{-3}$ & $3 \times 10^{-3}$ & $6 \times 10^{-3}$ \\
0.5 & $6 \times 10^{-3}$ & $6 \times 10^{-3}$ & $2 \times 10^{-3}$ & $1 \times 10^{-3}$ & $4 \times 10^{-3}$ & $9 \times 10^{-3}$ & $10^{-2}$ \\
0.7 & $10^{-2}$ & $10^{-2}$ & $10^{-2}$ & $9.9 \times 10^{-3}$ & $2 \times 10^{-2}$ & $4 \times 10^{-2}$ & $6 \times 10^{-2}$ \\
0.8 & $2 \times 10^{-2}$ & $2 \times 10^{-2}$ & $2 \times 10^{-2}$ & $2 \times 10^{-2}$ & $4 \times 10^{-2}$ & $7 \times 10^{-2}$ & 0.1106 \\
0.9 & $2 \times 10^{-2}$ & $3 \times 10^{-2}$ & $3 \times 10^{-2}$ & $4 \times 10^{-2}$ & $8 \times 10^{-2}$ & 0.12594 & 0.1862 \\
\hline
\end{tabular}

for $x<t$. Taking only two-term approximation for $u$ we have

$$
\begin{aligned}
u= & u_{0}+u_{1}=x-\frac{4}{45} t^{6}-\frac{4}{3} t^{4} x^{2}+\frac{4}{3} t^{4} x-\frac{1}{3} t^{4}-t^{2} x^{2}+t^{2} x \quad \text { for } x>t \\
u= & x-\frac{8}{15} t^{5} x+\frac{4}{3} t^{4} x-\frac{16}{9} t^{3} x^{3}-\frac{4}{3} t^{3} x+\frac{4}{3} t^{2} x^{4} \\
& +t^{2} x^{2}+t^{2} x-\frac{8}{15} t x^{5}-\frac{4}{3} t x^{3}+\frac{4}{45} x^{6}+\frac{1}{3} x^{4},
\end{aligned}
$$

for $x<t$. The absolute errors between the exact and two-term approximation of the series solution for some values of $(x, t) \in[0,1] \times[0,1]$ are shown in Table 2 . A higher accuracy level can be attained by evaluating some more terms.

Example 2.4. Now we consider the problem

$$
\begin{gathered}
u_{t t}-u_{x x}=u_{x}+u_{t}-2 x-2, \\
u(x, 0)=x^{2}, \quad u_{t}(x, 0)=0, \quad u(0, t)=\sin ^{2} t .
\end{gathered}
$$

It is easy to show that the HPM can not be applied if $L$ is taken as $u_{t t}$. The exact solution is

$$
\begin{aligned}
u & =x^{2}+\sin ^{2}(t-x) \quad \text { for } x<t \\
& =x^{2} \quad \text { for } x \geq t
\end{aligned}
$$

We construct the homotopy

$$
L u-L v_{0}+p\left[L v_{0}-u_{x}-u_{t}+2 x+2\right]=0
$$


with $L=u_{t t}-u_{x x}$. Substituting (1.7) into $u$ and equating the coefficients of like powers of $p$, we get a system of linear equations

$$
\begin{gathered}
L u_{0}=0, \quad u_{0}(x, 0)=x^{2}, \quad u_{0 t}(x, 0)=0, \quad u_{0}(0, t)=\sin ^{2} t, \\
L u_{1}=u_{0 x}+u_{0 t}-2 x-2, \quad u_{1}(x, 0)=0, \quad u_{1 t}(x, 0)=0, \quad u_{1}(0, t)=0, \\
L u_{2}=u_{1 x}+u_{1 t}, \quad u_{2}(x, 0)=0, \quad u_{2 t}(x, 0)=0, \quad u_{2}(0, t)=0, \ldots
\end{gathered}
$$

Solving we obtain

$$
\begin{aligned}
u_{0} & =\frac{1}{2}\left((x+t)^{2}-(t-x)^{2}\right)+\sin ^{2}(t-x)=2 t x+\sin ^{2}(t-x) \quad \text { for } x<t, \\
u_{0} & =\frac{1}{2}\left((x+t)^{2}+(x-t)^{2}\right)=x^{2}+t^{2} \quad \text { for } x>t \\
L u_{1} & =u_{0 x}+u_{0 t}-2 x-2=2 t+2 x-2 x-2=2 t-2, \\
u_{1} & =\frac{1}{2} \int_{0}^{t-x} \int_{t-x-s}^{x+t-s}(2 s-2) d y d s+\frac{1}{2} \int_{t-x}^{t} \int_{x-t+s}^{x+t-s}(2 s-2) d y d s \\
& =t^{2} x-t x^{2}-2 t x+\frac{1}{3} x^{3}+x^{2} \quad \text { for } x<t, \\
L u_{1} & =u_{0 x}+u_{0 t}-2 x-2=2 t-2, \\
u_{1} & =\frac{1}{2} \int_{0}^{t} \int_{x-t+s}^{x+t-s}(2 s-2) d y d s=\frac{1}{3} t^{2}(t-3) \quad \text { for } x>t .
\end{aligned}
$$

Continuing we obtain

$$
\begin{aligned}
L u_{2} & =u_{1 x}+u_{1 t}=t^{2}-2 t x-2 t+x^{2}+2 x+2 t x-x^{2}-2 x=t^{2}-2 t \\
u_{2} & =\frac{1}{2} \int_{0}^{t-x} \int_{t-x-s}^{x+t-s}\left(s^{2}-2 s\right) d y d s+\frac{1}{2} \int_{t-x}^{t} \int_{x-t+s}^{x+t-s}\left(s^{2}-2 s\right) d y d s \\
& =\frac{7}{3} t^{3} x-\frac{5}{2} t^{2} x^{2}-5 t^{2} x+\frac{4}{3} t x^{3}+3 t x^{2}-\frac{1}{4} x^{4}-\frac{2}{3} x^{3} \quad \text { for } x<t \\
u_{2} & =\frac{1}{2} \int_{0}^{t} \int_{x-t+s}^{x+t-s}\left(s^{2}-2 s\right) d y d s=\frac{1}{12} t^{4}-\frac{1}{3} t^{3} \quad \text { for } x>t .
\end{aligned}
$$

Thus the three-term approximation is

$$
\begin{aligned}
& u=\sin ^{2}(t-x)+x^{2}+\frac{7}{3} t^{3} x-\frac{5}{2} t^{2} x^{2}-4 t^{2} x+\frac{4}{3} t x^{3}+2 t x^{2}-\frac{x^{4}}{4}-\frac{x^{3}}{3} \quad \text { for } x<t \\
& u=x^{2}+\frac{1}{12} t^{4} \quad \text { for } x>t
\end{aligned}
$$

and the error is less than $(1 / 12) t^{4}$ for $x>t$. 


\section{Conclusion}

Homotopy perturbation method has been successful for solving many linear and nonlinear wave-type problems. However, it has difficulties in dealing with initial boundary problems, namely, in including all initial and boundary conditions together into the process of homotopy perturbation and computation. Our main goal is to construct the homotopy perturbation scheme containing all initial and boundary conditions together. The goal is achieved by involving an auxiliary operator which includes both variables $x$ and $t$.

\section{References}

[1] P. J. Caudray, I. C. Eilbeck, and J. D. Gibbon, "The sine-Gordon equation as a model classical field theory," Nuovo Cimento, vol. 25, pp. 497-511, 1975.

[2] R. K. Dodd, I. C. Eilbeck, and J. D. Gibbon, Solitons and Nonlinear Wave Equations, Academic, London, UK, 1982.

[3] S. J. Liao, On the proposed homotopy analysis technique for nonlinear problems and its applications, Ph.D. dissertation, Shangai Jio Tong University, Shangai, China, 1992.

[4] J. H. He, "Variational iteration method-a kind of non-linear technique: some examples," International Journal of Non-Linear Mechanics, vol. 34, pp. 699-708, 1999.

[5] J. H. He, "A coupling method of a homotopy technique and a perturbation technique for non-linear problems," International Journal of Non-Linear Mechanics, vol. 35, no. 1, pp. 37-43, 2000.

[6] J. H. He, "Application of homotopy perturbation method to nonlinear wave equations," Caos, Solitons E Fractals, vol. 26, pp. 695-700, 2005.

[7] J. H. He, Non-Perturbative Methods for Strongly Nonlinear Problems, Die Deutsche Bibliothek, Leipzig, Germany, 2006.

[8] J. H. He, "New interpretation of homotopy perturbation method," International Journal of Modern Physics B, vol. 20, no. 18, pp. 2561-2568, 2006.

[9] M.S. H. Chowdhury and I. Hashim, "Application of homotopy-perturbation method to Klein-Gordon and sine-Gordon equations," Chaos, Solitons E Fractals, vol. 39, no. 4, pp. 1928-1935, 2009.

[10] M. J. Ablowitz, B. M. Herbst, and C. Schober, "Homotopy perturbation method and axisymmetric flow over a stretching sheet," International Journal of Nonlinear Sciences and Numerical Simulation, vol. 7, no. 4, pp. 399-406, 2006.

[11] M. E. Berberler and A. Yildirim, "He's homotopy perturbation method for solving the shock wave equation," Applicable Analysis, vol. 88, no. 7, pp. 997-1004, 2009.

[12] L. Cveticanin, "The homotopy-perturbation method applied for solving complex-valued differential equations with strong cubic nonlinearity," Journal of Sound and Vibration, vol. 285, no. 4-5, pp. 11711179, 2005.

[13] D. D. Ganji and A. Rajabi, "Assessment of homotopy-perturbation and perturbation methods in heat radiation equation," International Communications in Heat and Mass Transfer, vol. 33, pp. 391-400, 2006.

[14] D. D. Ganji and A. Sadighi, "Application of homotopy-perturbation and variational iteration methods to nonlinear heat transfer and porous media equations," Journal of Computational and Applied Mathematics, vol. 207, no. 1, pp. 24-34, 2007.

[15] G. Adomian, "A review of the decomposition method in applied mathematics," Journal of Mathematical Analysis and Applications, vol. 135, no. 2, pp. 501-544, 1988.

[16] G. Adomian, Nonlinear Stochastic Systems and Applications to Physics, vol. 46 of Mathematics and its Applications, Kluwer Academic Publishers, Dordrecht, Germany, 1989.

[17] A. M. Wazwaz, "A comparison between Adomian decomposition method and Taylor series method in the series solutions," Applied Mathematics and Computation, vol. 97, no. 1, pp. 37-44, 1998.

[18] D. Kaya, "A numerical solution of the sine-Gordon equation using the modified decomposition method," Applied Mathematics and Computation, vol. 143, no. 2-3, pp. 309-317, 2003.

[19] S. M. El-Sayed, "The decomposition method for studying the Klein-Gordon equation," Chaos, Solitons E Fractals, vol. 18, no. 5, pp. 1025-1030, 2003.

[20] A. M. Wazwaz and A. Gorguis, "Exact solutions for heat-like and wave-like equations with variable coefficients," Applied Mathematics and Computation, vol. 149, no. 1, pp. 15-29, 2004.

[21] W. A. Strauss, Partial Differential Equations, John Wiley \& Sons, New York, NY, USA, 1992. 


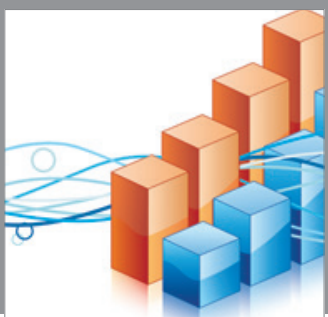

Advances in

Operations Research

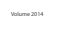

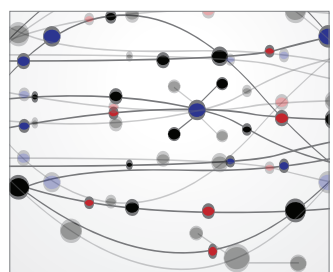

\section{The Scientific} World Journal
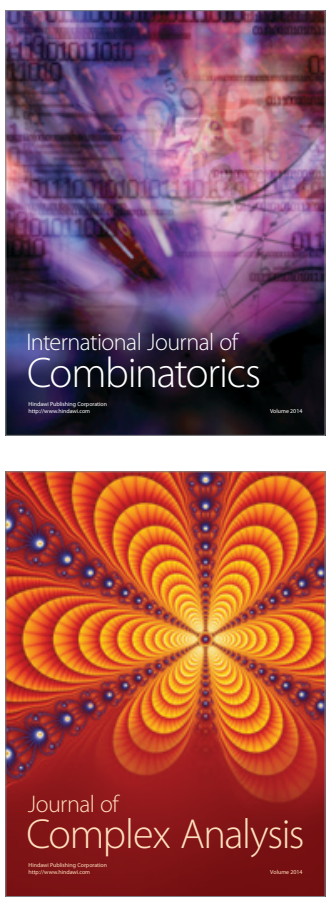

International Journal of

Mathematics and

Mathematical

Sciences
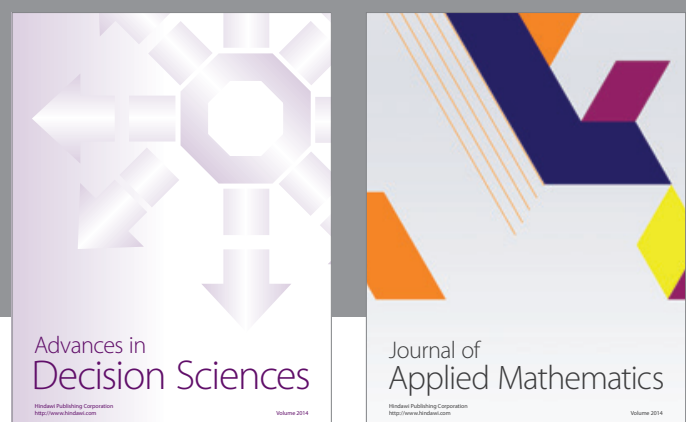

Journal of

Applied Mathematics
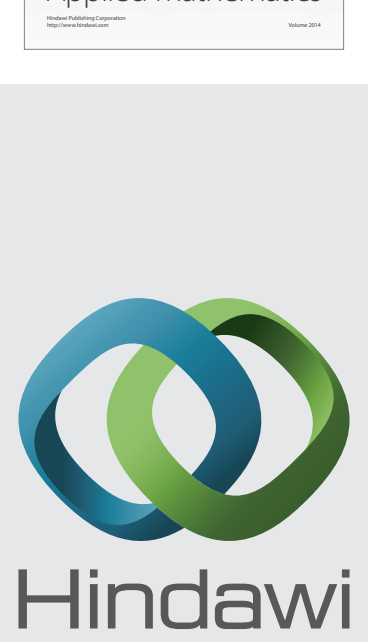

Submit your manuscripts at http://www.hindawi.com
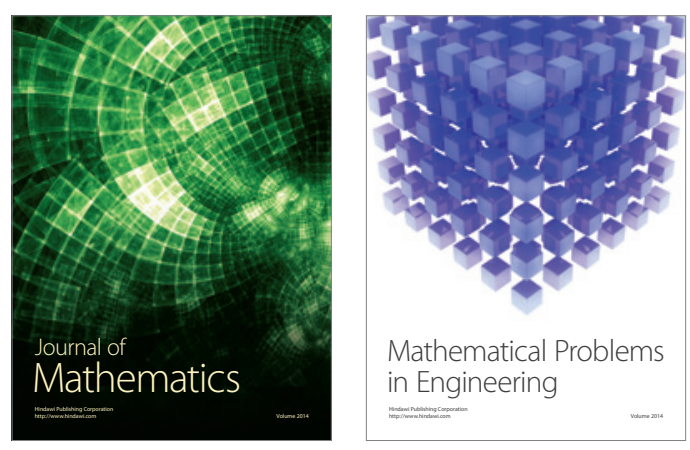

Mathematical Problems in Engineering
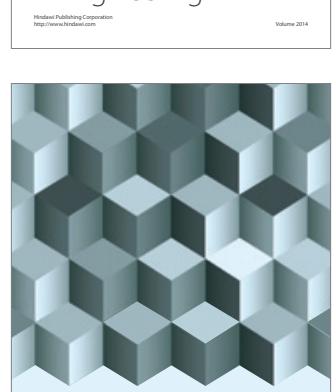

Journal of

Function Spaces
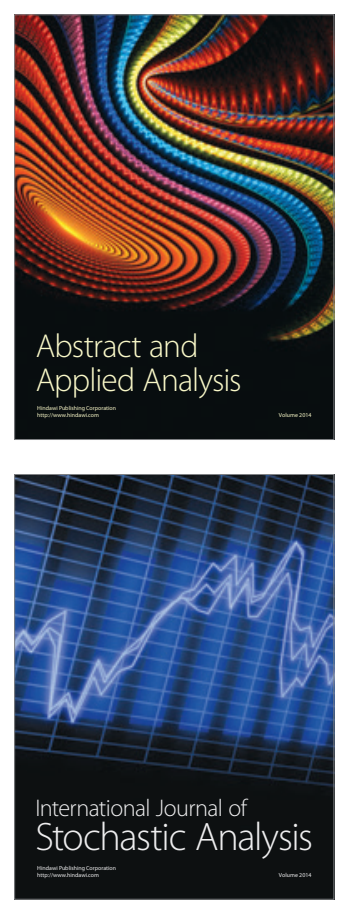

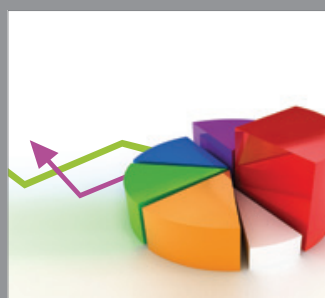

ournal of

Probability and Statistics

Promensencen
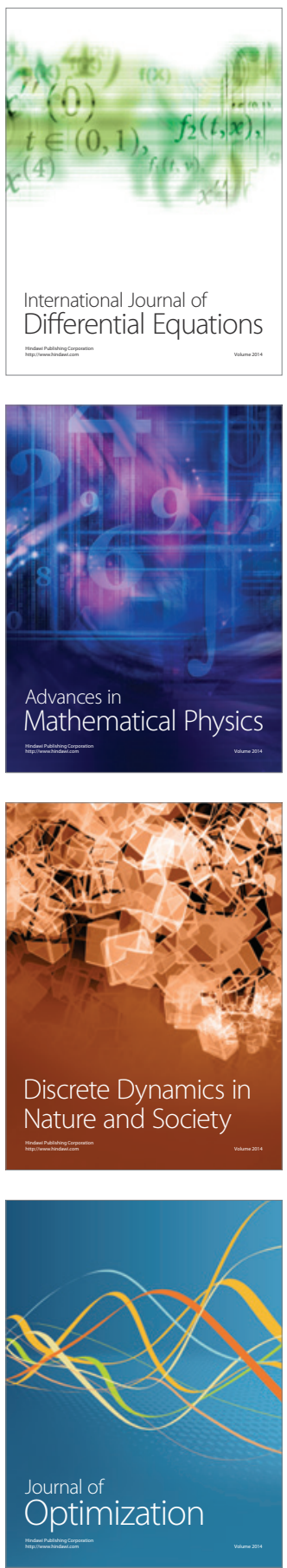